\title{
Efficiency of Speed Advisory Boundary fINder (SABIN) strategy for GLOSA using ITS-G5
}

\author{
Mouna Karoui, Antonio Freitas, Gérard Chalhoub \\ LIMOS-CNRS - University Clermont Auvergne \\ \{mouna.karoui, antonio.freitas, gerard.chalhoub\}@uca.fr
}

\begin{abstract}
Cooperative Intelligent Transportation Systems (CITS) enhance road safety and traffic management. GLOSA (Green Light Optimized Speed Advisory) is an ITS service that helps reduce traffic jam. In this paper, we present a strategy algorithm for GLOSA called Speed Advisory Boundary fINder (SABIN) that allows vehicles to pass a traffic light when it is green as fast as possible or as slow as possible. We evaluated SABIN in terms of average stoppage time, travel time, average number of stopped vehicles and fuel consumption. We also evaluate the impact of network performance on SABIN.
\end{abstract}

Index Terms-C-ITS, GLOSA, ETSI- ITS-G5, Average stoppage time, packet loss.

\section{INTRODUCTION}

Intelligent transportation systems (ITS) are currently receiving a great interest due to their important contribution in decreasing road hazards and traffic jam. Traffic congestion is one of the major problems faced by modern transportation systems. This issue can be solved through C-ITS services that are capable of enhancing road safety. ITS services are classified into three categories: safety, traffic efficiency and infotainment. Safety applications include collision avoidance and accident notification services. Traffic efficiency applications are designed to provide driving assistance, and useful information about road conditions. The aim of infotainment services is to make driving experience more comfortable and less sophisticated.

Green light Optimized Speed Advisory (GLOSA) is one of the efficiency traffic applications that play a crucial role to improve the fluidity of roads. GLOSA is able to determine adequate speed according to traffic lights signal status. Thus, driver can be advised to change his speed in order to pass the next traffic light during the green phase. GLOSA can also give the driver speed that enables him to cross a set of consecutive traffic lights during green phases. This kind of speed advisory service can significantly improve traffic congestion and unnecessary acceleration will be avoided. Consequently, fuel consumption and gas emissions will be decreased. In this context, several communication technologies are used in order to ensure the real implementation of this ITS service. Particularly, the use of Cooperative Intelligent Transportation Systems (C-ITS) will be required. C-ITSs enable vehicles to communicate with each other, with roadside infrastructure as well as traffic signals. These systems use different communication standards such as IEEE 802.11p, ETSI ITS-G5, and other cellular communication such as LTE. In this paper, we will use ITS-G5 architecture for communication between vehicles and roadside units.

Many European projects are working in this domain. They basically use real implementations and Field Operational Tests (FOTs) to evaluate the performance of this kind of services. In particular, C-ROADS is a European C-ITS deployment project which goal is to develop and to assess the scalability of C-ITS solutions. One of the major challenges for this project is to properly define realistic scenarios to evaluate performance of C-ITS services considering real use cases. In this paper, we propose a Speed Advisory Boundary fINder strategy (SABIN) for GLOSA. We will validate our algorithm using a coupled simulation environment with the definition of a real-life inspired scenario.

The main objective of this work is to study the efficiency of SABIN in terms of different criteria related to traffic fluidity. We also study the impact of ETSI ITS-G5 network performance on the efficiency of SABIN.

The remainder of this paper is organized as follows. First, in section II, we present important contributions in related work about GLOSA and different performance evaluation of this application. In section III, we describe SABIN. Simulation framework and test scenarios are described in section IV-B. In section $\mathrm{V}$, we study the efficiency of SABIN with regards to network congestion. Finally, we conclude the paper in section VI and discuss some perspectives for our future work.

\section{RELATED WORK}

In this section we present research work that studied performance of GLOSA. Authors in [1] proposed a GLOSA algorithm. They evaluated their algorithm using the integrated simulation platform VSimRTI according to three aspects. At the beginning, they observed the influence of activation distance for GLOSA on overall performance. Then, they simulated the influence of penetration rate on GLOSA. Finally, they assessed GLOSA by varying traffic densities. Their findings show that GLOSA has positive effects on all performance metrics. On the other hand, the main limitation of this study is that during a green phase, the maximum authorized speed is given without any verification if the speed enables driver to pass green phase or not. Compared to without GLOSA, they obtain an improvement of average stoppage time behind traffic light of about $89 \%$. 
In [2], authors studied GLOSA in the context of DRIVE $\mathrm{C} 2 \mathrm{X}$ project by simulation. This paper focused on predicting traffic efficiency effects of GLOSA. They used SUMO as traffic simulation environment coupled with a communication model based on IEEE802.11e standard. They measured journey duration and $\mathrm{CO} 2$ emissions against communication range. They found that communication range has a direct impact on GLOSA performance. Their findings showed that a communication range of $1000 \mathrm{~m}$ gives vehicles the needed information about traffic light state. On the other hand, authors did not study the impact of packet loss on GLOSA performance.

Paper [3] presents an implementation of GLOSA using a simulation framework that combines a traffic simulator and a network simulator with multi user driving platform. This study focuses on emphasizing human factor which relies on different driver behaviors in order to obtain realistic evaluation of GLOSA. Authors measured stopping time, travel time, $\mathrm{CO} 2$ emissions, and average speed considering various penetration rates. Their results show that human behavior and his acceptance to speed advice can decrease performance of GLOSA when only $10 \%$ of vehicles are equipped. However, increasing penetration rate can improve the situation and reduce driver behavior impact. In this study, authors didn't precise network conditions, they only concentrate their interests on driver behavior and his effect on GLOSA performance.

Authors in [4] presented a multi-segment GLOSA approach which consists of speed adaptation according to a sequence of traffic lights. Advisory speed is given according to the chosen preferences like fuel consumption minimization or travel time reduction. A Genetic Algorithm (GA) is used in this context to solve the problem of searching the optimal set of speeds through a huge number of possibilities for solutions. In this paper, authors introduced three approaches: GA-based optimization with minimization of fuel consumption (Approach FUEL), GA-based optimization with minimization of traveling time (Approach TIME) and single segment fuel optimization (Approach FUEL-IND). Authors compared single segment and multi-segment GLOSA algorithms. Results showed that keeping a constant speed significantly improves fuel consumption and gas emissions. In addition, obtained results with multi-segment GLOSA have better performance than single segment GLOSA algorithm. Simulation scenario was restricted to free flow traffic conditions. Authors did not show the efficiency of Genetic Algorithm in high density scenarios. Also, heuristic algorithms like GA takes a lot of calculation time and they did not give details about execution mode of GA algorithm.

Authors in [5] tested GLOSA using both simulation and real experimentation. They used algorithm presented in [1]. As a first step, they applied GLOSA only to one vehicle considering a ring traffic topology. Then, they tested the same scenario by varying vehicles number. In their study, they measured the gain of GLOSA in terms of CO2 emissions, travel time and waiting time. They compared their results with a system without GLOSA. Their findings showed that GLOSA have benefits on non equipped vehicles, especially with a penetration rate of 50
$\%$. On the other hand, authors studied GLOSA performance on ideal network without access delay or packet loss.

Paper [6] presented a real world GLOSA system assessment based on Field Operational Test (FOT) results of DRIVE C2X European project. They proposed a set of well established metrics for GLOSA technical evaluation. They classified their metrics according to different layers: network and transport, facilities and application layers. They measured End-to-End Delay (E2ED), Packet Delivery Ratio (PDR) and information distance. Their findings showed that RSUs (Road Side Units) location should be well chosen because it affects GLOSA performance in terms of communication distance. As a result, they found a maximum information distance of $644 \mathrm{~m}$. They noticed that every traffic light has specific characteristics in terms of median information distance and distribution of recorded values. Also, authors did not evaluate the impact of network performance on GLOSA efficiency.

\section{SPEED ADVISORY BOUNDARY FINDER (SABIN)}

In this section, we propose a speed estimation study and we describe SABIN algorithm. The idea is to propose an algorithm that calculates advisory speed boundaries for GLOSA application. According to a certain strategy, we can choose an advisory speed between two boundaries $v_{1}$ and $v_{2} . v_{1}$ is the calculated speed to pass at the start time of green phase. $v_{2}$ is the calculated speed to pass at the end time of the green phase. Using our proposition, drivers can pass traffic light with any speed between $v_{1}$ and $v_{2}$.

\section{A. Speed estimation study}

Figure 1 shows the evolution of velocity against time. We suppose that acceleration $a$ and deceleration decel are constant and we consider a uniformly varied motion.

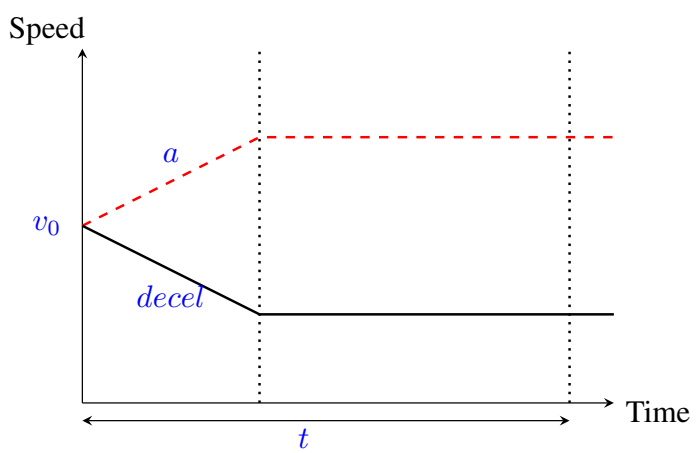

Fig. 1. Velocity Vs Time

The evolution of velocity is divided into two phases, a phase of acceleration or deceleration and a phase in which the speed becomes constant. After resolving a system of equations, we obtain equation 1 . This equation is determined according to a set of parameters:

- $d$ is the distance to the traffic light,

- $v_{0}$ is the initial speed,

- $t$ is the duration in which we want to calculate the target speed. 


$$
v_{\text {target }}(t)=a *\left(t-\sqrt{t^{2}-\frac{2\left(d-v_{0} * t\right)}{a}}\right)+v_{0}
$$

Equation (1) exists if the conditions below are verified: Acceleration case $a>0$ :

$$
\left\{\begin{array}{c}
v_{0}<\frac{d}{t} \\
a>\frac{2 *\left(d-v_{0} * t\right)}{t^{2}}
\end{array}\right.
$$

Deceleration case $a<0$ :

$$
\left\{\begin{array}{c}
v_{0}>\frac{d}{t} \\
a<\frac{2 *\left(d-v_{0} * t\right)}{t^{2}}
\end{array}\right.
$$

SABIN algorithm has the following input parameters: Green phase duration, distance to traffic light, current time and initial speed. At the beginning, it calculates $t_{\text {pmax }}$ and compares its value with the end of the green phase. This test enables us to define cases in which we need to repeat the algorithm in order to get an adequate advisory speed for the upcoming green phase.

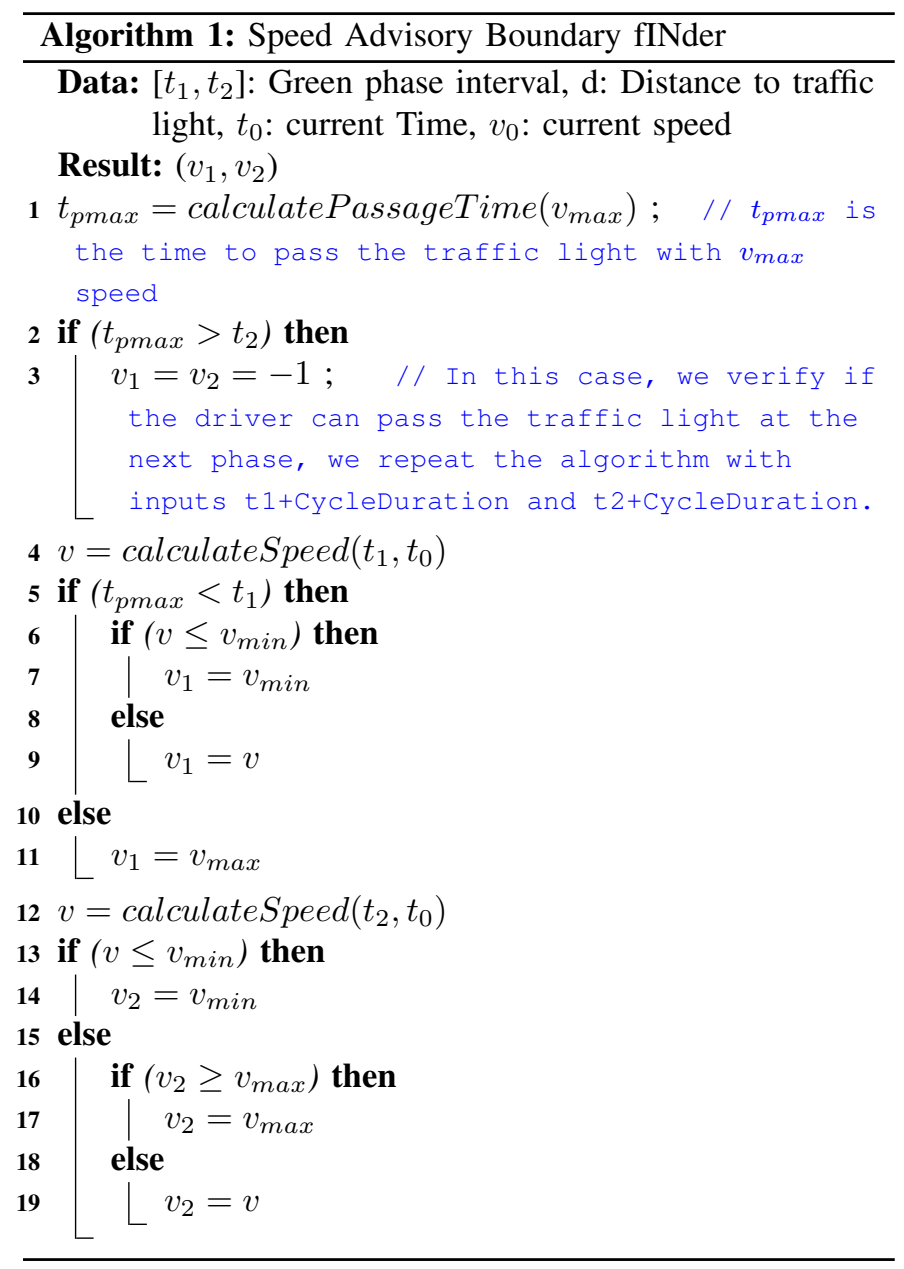

Figure 2 describes speed calculation process applying conditions in III-A and equation (1). $(v=-1)$ means that the function returns no solution. This case is considered in SABIN when faced with scenarios that cannot allow a vehicle to cross traffic light while it is green.

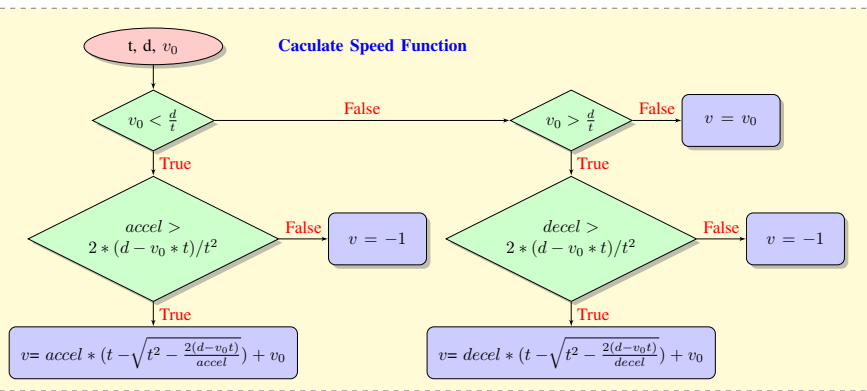

Fig. 2. Calculate Speed function

\section{PERFormance STUdy OF SABIN}

In this section we describe the simulation platform that we used for our evaluation of SABIN.

\section{A. Artery simulation model}

We used Artery framework ${ }^{1}$ to simulate SABIN and evaluate its performance. Artery framework is an extended version of VEINS (Vehicles In network Simulation). VEINS framework couples the traffic Simulation of Urban Mobility (SUMO) with the discrete event simulation tool OMNET++. SUMO establishes a connection with VEINS using Traffic Control Interface (TraCI) protocol. TraCI is based on a client/server TCP architecture. It gives access to the running road traffic simulation in order to get different parameters like speed and fuel consumption of simulated objects such as vehicles.

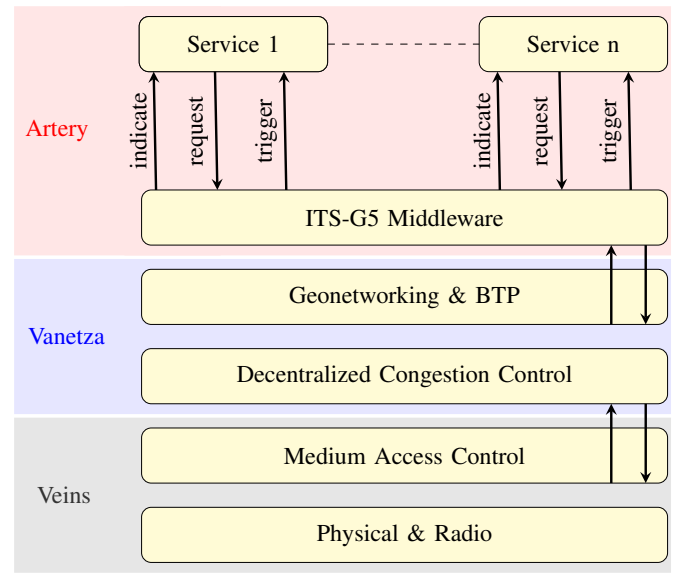

Fig. 3. Artery architecture

We have chosen Artery because it is an open source project that provides a realistic model of ETSI ITS-G5 reference architecture as shown in figure 3. It uses IEEE 802.11p VEINS and INET components in order to model V2X communications. Artery introduces an enhanced version of Medium Access Control (MAC) that generates channel load measurements used as input for Decentralized Congestion Control

${ }^{1}$ https://github.com/riebl/artery 
algorithm (DCC) provided by ETSI specifications which is implemented in Vanetza. It also provides a full implementation of Cooperative awareness message (CAM) and Decentralized Environmental Notification (DENM) basic services.

This simulation tool is also characterized by its flexibility in terms of development. It is built using CMake cross-platform tools so that it provides an easy way to add external libraries and other functionalities at the different layers [7].

\section{B. Simulation scenario description}

In this section, we consider a two-lane highway scenario as shown in figure 4. Table I describes simulation parameters. We tested SABIN in ideal conditions where there is no network congestion and in case of a $100 \%$ of penetration rate. In our case, vehicles can change lanes according to SUMO lanechanging model, but they cannot overtake another vehicle.

TABLE I

SIMULATION PARAMETERS

\begin{tabular}{c||c}
\hline Acceleration & $1 \mathrm{~m} / \mathrm{s}^{2}$ \\
\hline \hline Deceleration & $2 \mathrm{~m} / \mathrm{s}^{2}$ \\
\hline \hline$v_{\max }, v_{0}$ & $50 \mathrm{~km} / \mathrm{h}$ \\
\hline \hline$v_{\min }$ & $20 \mathrm{~km} / \mathrm{h}$ \\
\hline \hline Vehicle type & Gasoline driven light duty vehicle, Euro norm 4 \\
\hline \hline Traffic light phases & Red $(30 \mathrm{~s})$, Green $(25 \mathrm{~s})$, Yellow $(5 \mathrm{~s})$ \\
\hline \hline Traffic topology & Two lane highway scenario \\
\hline
\end{tabular}

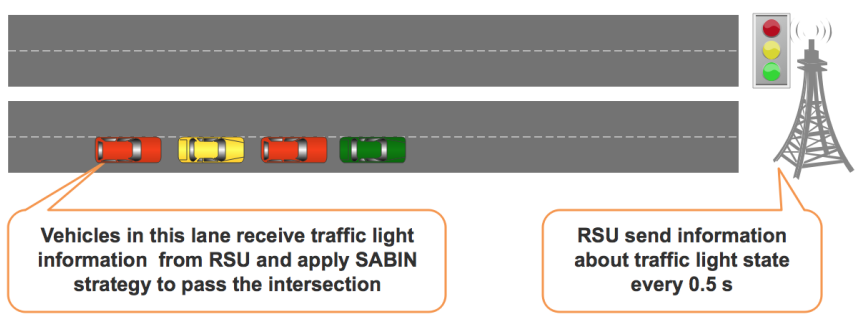

Fig. 4. Simulation scenario without network congestion.

We present a comparative study of SABIN and a scenario without GLOSA. We measured speed, fuel consumption, average stop time and stopped vehicles number at traffic light. Fuel consumption is measured according to SUMO models: HBEFA and PHEMlight.

HBEFA $^{2}$ model is based on the database application "HandBook Emission FActors for road transport" which contains information about polluant emissions and fuel consumption. This model depends on speed and acceleration or deceleration variation. PHEMlight ${ }^{3}$ (Passenger car \& Heavy duty Emission Model) is an instantaneous model included in SUMO.

In figure 5, speed and fuel consumption graphs against simulation time are presented. We observe that SABIN gives driver an advisory speed $\left(v_{1}\right)$ that enables him to pass traffic

\footnotetext{
${ }^{2}$ http://sumo.dlr.de/wiki/Models/Emissions/HBEFA-based

${ }^{3}$ http://sumo.dlr.de/wiki/Models/Emissions/PHEMlight
}

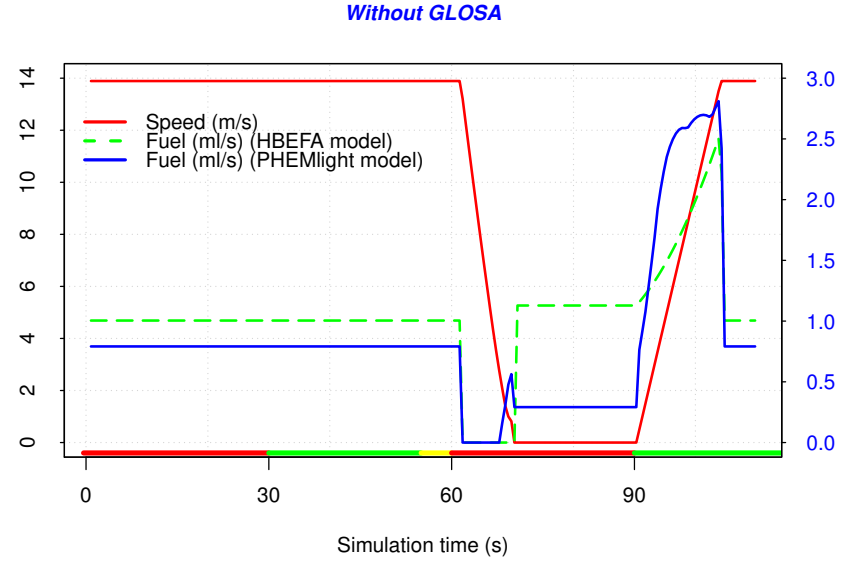

With SABIN strategy for GLOSA

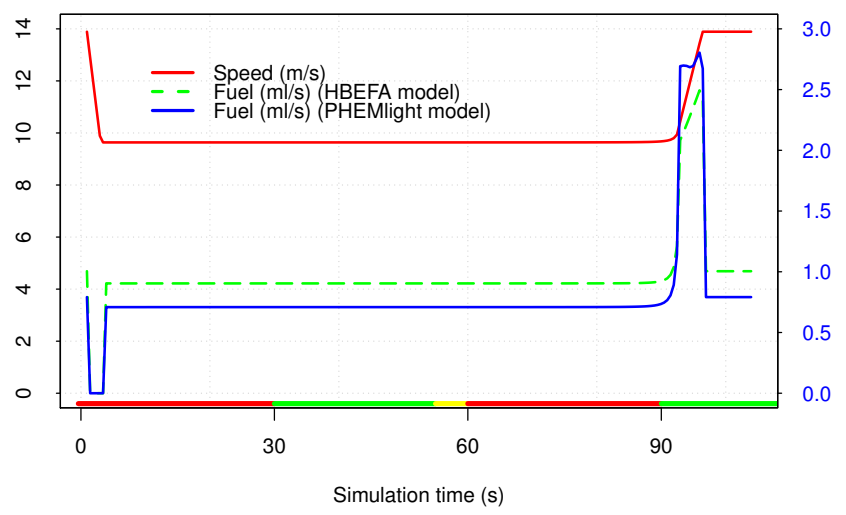

Fig. 5. Fuel consumption and speed comparison.

light at the start of the upcoming green phase at about $93 \mathrm{~s}$. We introduced a delay of 3 seconds to avoid deceleration of the vehicle introduced by SUMO traffic simulator when traffic light switches from red to green.

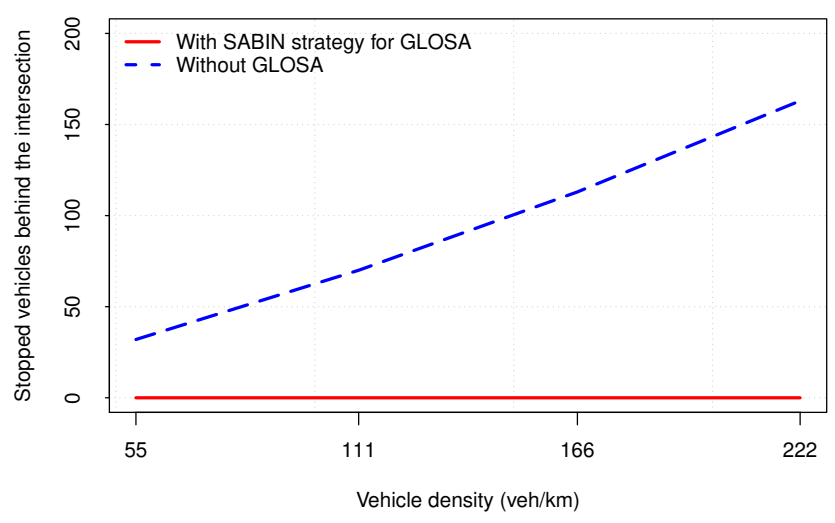

Fig. 6. Stopped vehicles number behind the traffic light Vs vehicles density. 
Figure 7 presents, a comparative study between SABIN and a scenario without GLOSA in terms of stopped vehicles number behind the traffic light. We observe that with SABIN strategy, vehicles adapt their speed to pass the traffic light in the current green phase or in the upcoming one.

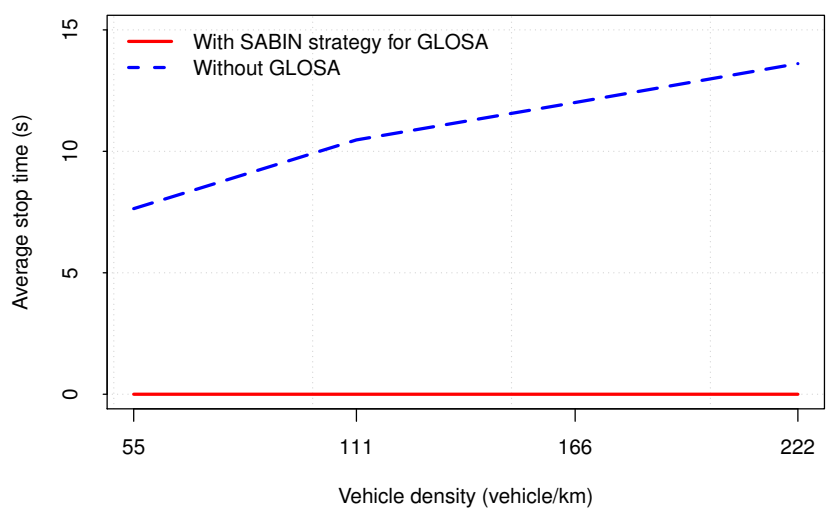

Fig. 7. Average stoppage time behind the traffic light Vs vehicles density.

In figure 8, fuel consumption is measured using HBEFA model against penetration rate variation. We observed that with $0 \%$ of penetration rate, vehicles consume about $1 \mathrm{ml} / \mathrm{s}$ during a total distance of about $1.8 \mathrm{~km}$. In this case vehicles did not adapt their speed, and they stop behind the traffic light. When SABIN is applied, we get a fuel consumption of about $0.75 \mathrm{ml} / \mathrm{s}$ when all vehicles are equipped $(100 \%)$. Thus, SABIN reduces fuel consumption by keeping speed as constant as possible and avoiding stops that cause unnecessary acceleration and deceleration. As a result, SABIN is able to save $25 \%$ of fuel passing for a penetration rate of $100 \%$.

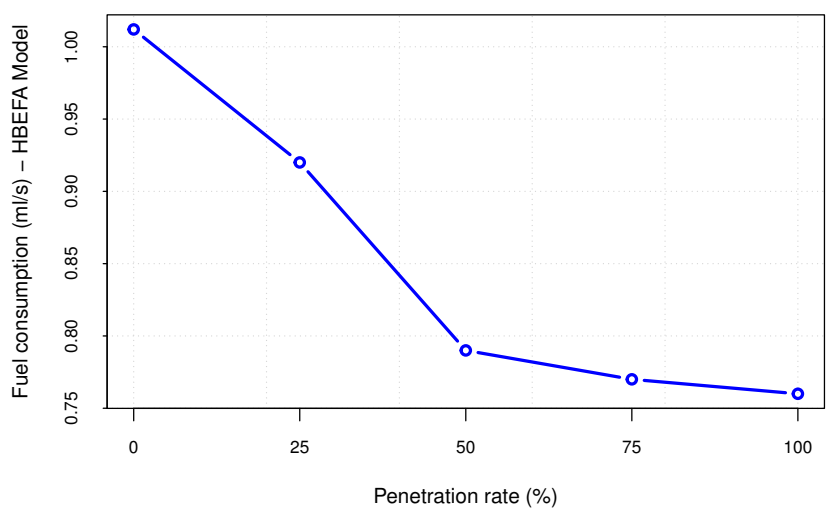

Fig. 8. Fuel consumption Vs penetration rate
TABLE II

COMMUNICATION PARAMETERS

\begin{tabular}{c||c}
\hline Transmission range & $\approx 1280 \mathrm{~m}$ \\
\hline \hline Propagation model & Free space \\
\hline \hline Penetration rate & $100 \%$
\end{tabular}

\section{IMPACT OF NETWORK PERFORMANCES ON SABIN STRATEGY FOR GLOSA}

In this section, we highlight the impact of network performance on SABIN. We create congestion in the network by adding stopped vehicles disseminating periodical Cooperative Awareness Messages (CAM) every 0.01 second as shown in figure 9. According to ETSI specifications, CAM messages can be sent using a sending frequency between $10 \mathrm{~Hz}$ and $100 \mathrm{~Hz}$. We chose $100 \mathrm{~Hz}$ since we are interested in creating critical network congestion scenario for evaluation purposes. Table II presents communication parameters used in our network scenario. Stopped vehicles number is variable from 50 to 229 in a road of 900 meters (Distance to traffic light), which is equivalent to a traffic density between $55 \mathrm{veh} / \mathrm{km}-222$ $\mathrm{veh} / \mathrm{km}$. We compared the obtained results with the ideal case in previous section.

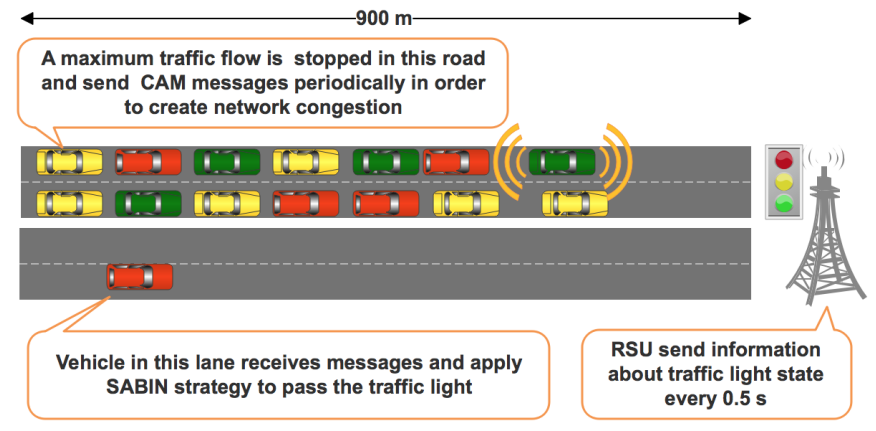

Fig. 9. Stopped vehicles density variation scenario.

In this section two network metrics are defined:

- Packet loss rate is measured using formula (2)

$$
P_{\text {loss }}=\frac{\text { totalLostPackets }}{\text { totalLostPackets+totalReceivedPackets }}
$$

- MAC busy time is the fraction of time that the Medium Access layer was sensed busy.

Figure 10 presents packet loss rate evolution and busy time against stopped vehicles number for the RSU node. We observe an increase of packet loss rate and MAC busy time when increasing stopped vehicles density. It means that sending capability of RSU is decreasing every time we increase stopped vehicles density.

In figure 11, we measure travel time to traffic light against stopped vehicles density.

Results show that average travel time to traffic light is not affected by packet loss rate even in case of an augmentation of 


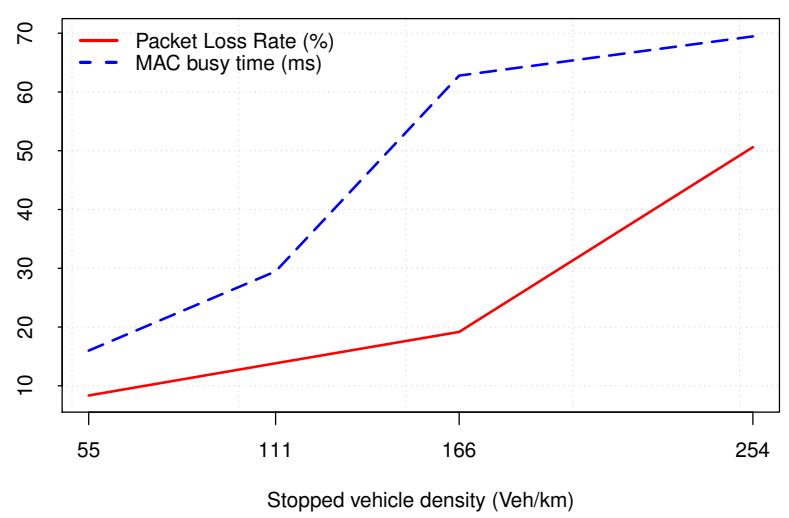

Fig. 10. RSU packet loss rate and MAC busy time

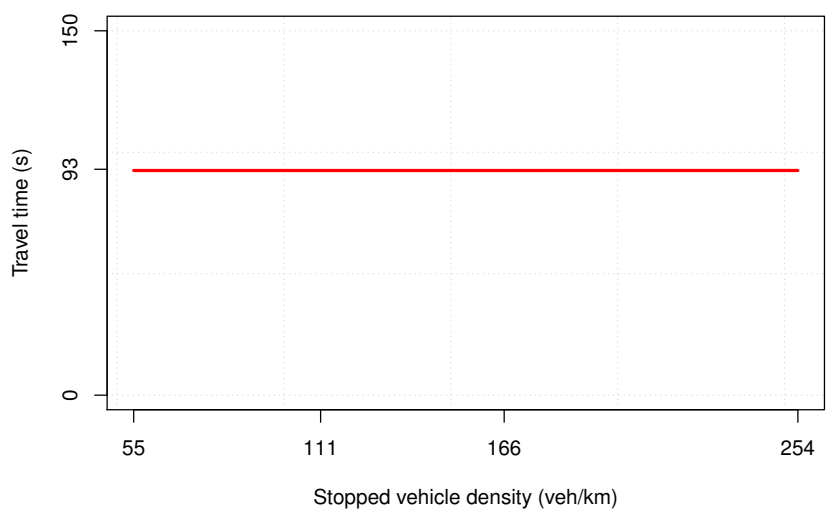

Fig. 11. Travel time to the traffic light.

about 50\%. Also average stop time remains 0 second compared to previous results. It is an expected result, because the speed adaptation depend strongly on the first message reception time.

\section{CONCLUSION AND FUTURE WORK}

In this paper, we proposed SABIN strategy for GLOSA that calculates boundary speeds to reach the traffic light using two approaches: arrive at the start of the green phase or arrive at the end of the green phase. We implemented our algorithm on Artery framework that provides realistic simulations of ETSI ITS-G5 protocols and services like CAM and DENM.

We tested SABIN using the first approach which is passing the traffic light as fast as possible. Then, we compared fuel consumption, speed and average stoppage time between SABIN and a scenario without GLOSA service. Results show that vehicles were able to avoid stopping at traffic light for different traffic densities. As a result, fuel consumption is reduced significantly by avoiding unnecessary deceleration and acceleration. Our results showed an improvement of fuel consumption of about $25 \%$ for a $100 \%$ of penetration rate.
We also evaluated the impact of network performance on SABIN through defining stopped vehicles density variation scenario where we set a number of stopped vehicles broadcasting CAM messages every 0.01 second. We measured packet loss and busy time in MAC layer for different stopped vehicles densities. We observed that the augmentation of packet loss of about $50 \%$ did not affect performance of SABIN strategy in terms of travel time and average stop time.

In our future work, we will focus on studying SABIN algorithm using different traffic topologies such as urban areas. We will propose a more realistic network scenario in terms of propagation model and communication range.

\section{REFERENCES}

[1] K. Katsaros, R. Kernchen, M. Dianati, and D. Rieck, "Performance study of a green light optimized speed advisory (glosa) application using an integrated cooperative its simulation platform," in Wireless Communications and Mobile Computing Conference (IWCMC), 2011 7th International. IEEE, 2011, pp. 918-923.

[2] D. Krajzewicz, L. Bieker, and J. Erdmann, "Preparing simulative evaluation of the glosa application," in Proceedings CD ROM 19th ITS World Congress 2012 Wien, Österreich, Paper ID: EU-00630, 2012.

[3] K. Gajananan, S. Sontisirikit, J. Zhang, M. Miska, E. Chung, S. Guha, and $\mathrm{H}$. Prendinger, "A cooperative its study on green light optimisation using an integrated traffic, driving, and communication simulator," Proceedings 36th-ATRF, 2013.

[4] M. Seredynski, W. Mazurczyk, and D. Khadraoui, "Multi-segment green light optimal speed advisory," in Parallel and Distributed Processing Symposium Workshops \& PhD Forum (IPDPSW), 2013 IEEE 27th International. IEEE, 2013, pp. 459-465.

[5] M.-A. Lebre, F. L. Mouël, E. Ménard, A. Garnault, B. Bradaï, and V. Picron, "Real scenario and simulations on glosa traffic light system for reduced $\mathrm{co} 2$ emissions, waiting time and travel time," arXiv preprint arXiv:1506.01965, 2015

[6] R. Stahlmann, M. Möller, A. Brauer, R. German, and D. Eckhoff, "Technical evaluation of glosa systems and results from the field," in Vehicular Networking Conference (VNC), 2016 IEEE. IEEE, 2016, pp. $1-8$.

[7] R. Riebl, H.-J. Günther, C. Facchi, and L. Wolf, "Artery: Extending veins for vanet applications," in Models and Technologies for Intelligent Transportation Systems (MT-ITS), 2015 International Conference on. IEEE, 2015, pp. 450-456. 